\title{
The Association between Applied Health Policies and Probability of Premature Death in Bulgaria
}

\author{
Mariela Stefanova Kamburova ${ }^{1}$ \\ Medical University \\ Stela Ludmilova Georgieva ${ }^{2}$ \\ Medical University \\ Manolya $\mathrm{Un}^{3}$ \\ Medical University
}

\begin{abstract}
Non-communicable diseases (NCDs) are estimated to account for $94 \%$ of all deaths in Bulgaria. The duty of a national health system is to implement health policies for reducing the negative consequences of premature death (between the ages of 30-70) due to NCDs for public health. Analyzing the association between the number of health policies applied in response to NCDs and the likelihood of premature death in Bulgaria involved assessing the situation in Bulgaria compared with other member states of the European Union (EU). Between October and December 2015, a comprehensive search of the literature on the subject of the study was conducted. Data were processed statistically and illustrated with tables and graphs. For 2014, the probability of premature death from NCDs (cardiovascular diseases, cancer, chronic lung diseases, or diabetes) in Bulgaria was 24\%; this was the highest level of any EU country. Bulgaria has the lowest number of applied health policies (two) in comparison with other EU member states (i.e., nine in Lithuania). They are only related to evidence-based national guidelines, protocols, and standards for managing major NCDs using a primary care approach and a national, population-based cancer registry. Implementing effective health policies in Bulgaria is essential for improving public health and reducing the probability of premature death in the country.
\end{abstract}

\section{Keywords}

Premature death $\bullet$ Non-communicable diseases $\bullet$ Health policy $\bullet$ Public health $\bullet$ Risk factors

\footnotetext{
1 Correspondence to: Mariela Stefanova Kamburova, Department of Public Health Sciences, Faculty of Public Health, Medical University, Pleven, Bulgaria Email: mariela_kamburova@yahoo.com

2 Department of Public Health Sciences, Faculty of Public Health, Medical University, Pleven, Bulgaria.

Email: georgieva_sl@yahoo.com

3 Faculty of Medicine, Medical University, Pleven, Bulgaria. Email: manolyaun@gmail.com

Citation: Kamburova, M. S., Georgieva, S. L., \& Un, M. (2017). The association between applied health policies and probability of premature death in Bulgaria. Sanitas Magisterium, 3, 47-52. http://dx.doi.org/10.12738/SM.2017.1.0027
} 
Health policies are a system of long-term policy decisions that a country adopts in the field of health protection. According to Borisov (1998), health policies reflect the health needs of the population and the health resources and values of a society.

One of the priorities of a health policy in developed countries is to expand applying its population strategy primarily for prevention. Such efforts focus on preventing the leading socially significant diseases and their related behavioral risk factors (Grancharova \& Alexandrova-Jankulovska, 2015).

Chronic NCDs, defined as associated with lifestyle diseases, are the leading cause of death worldwide. About 36 of the 57 million deaths worldwide in 2008 (63\%) were due to NCDs (World Health Organization [WHO], 2010; 2015). The most significant among them (cardiovascular diseases, cancer, chronic respiratory diseases and diabetes) accounted for approximately $80 \%$ of NCD deaths. These diseases occur mainly due to four modifiable behavioral risk factors: tobacco smoking (raises blood pressure), obesity, and alcohol abuse (Bonita et al., 2013).

In the EU, NCDs are the leading cause of death; more than $90 \%$ of deaths from chronic diseases are due to cardiovascular diseases, cancer, chronic respiratory diseases, and diabetes (WHO, 2014a).

An obligation of the health system of each country is to implement policies for reducing the negative public health consequences of these diseases and related risk factors.

\section{Purpose}

The purpose of the study is to analyze the association between the number of health policies applied in Bulgaria in response to non-communicable diseases and the likelihood of premature death, as well as to assess the situation in Bulgaria by comparing it with the other member states of the EU.

\section{Methods}

From October to December 2015, a comprehensive search of the literature on the study's subject was conducted. The search's inclusion criteria were: articles in English or original works in Bulgarian published in the last five years. The exclusion criteria were literary reviews and articles for evaluating the effectiveness of medical technologies. The search was conducted in PubMed and reviewed 11 full-text articles with the keyword "Health policy against non-communicable diseases."

Reports from WHO on the global status for chronic non-communicable diseases (2010, 2014a) and health (2015) were used as information sources. Data were processed statistically and illustrated with tables and graphs. 


\section{Determinants}

Health policies are defined as: decisions, strategies, or actions taken to achieve a specific health purpose in society.

Premature death is defined as: dying between the ages of 30-70, or dying under 70 .

\section{Results and Discussion}

Chronic diseases kill 38 million people each year. Over 40\% (16 million) are premature and occur under the age of 70. Leading causes of death due to NCDs are cardiovascular diseases, cancer, respiratory diseases, and diabetes.

In the structure of causes of death in Bulgaria for 2014 (Figure 1), the leading positions are: cardiovascular diseases $(65.9 \%)$, cancers $(16.6 \%)$, diseases of the digestive system (3.5\%), and others (14.0\%). NCDs are estimated to account for $94 \%$ of all deaths in Bulgaria.

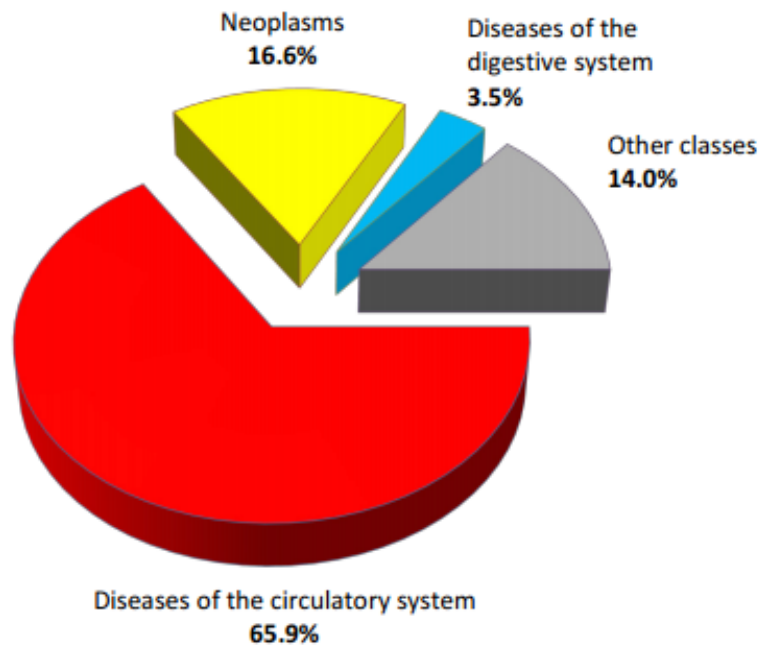

Figure 1. Mortality by Cause in 2014.

Source: National Center of Public Health and Analyses (2015).

A significant percentage of these deaths are premature. The likelihood of premature death from cardiovascular diseases, cancer, chronic respiratory diseases, or diabetes varies in different European countries from 9-24\%. The lowest level is reported in Cyprus (9\%) and the highest in Bulgaria, Hungary, and Latvia (24\%).

Behavioral risk factors, particularly smoking, alcohol abuse, high blood pressure, and obesity, are a major cause of NCDs. The frequency of its distribution among the adult population in Bulgaria is presented in Table 1. 
Table 1

Risk Constellation of Bulgarian Population

\begin{tabular}{lccc}
\hline Risk factor & men & Women & total \\
\hline Smoking & $48 \%$ & $31 \%$ & $39 \%$ \\
Alcohol & $17.9 \%$ & $5.3 \%$ & $11.4 \%$ \\
High blood pressure & $44.3 \%$ & $39.5 \%$ & $41.8 \%$ \\
Obesity & $23.1 \%$ & $24.3 \%$ & $23.7 \%$ \\
\hline
\end{tabular}

Source: (WHO, 2014c)

The most significant risk factor in the country is high blood pressure (41.8\%). Hypertension control in other EU countries is significantly more effective than in Bulgaria. The percentages of people with high blood pressure are $28.4 \%$ in Austria, $27.5 \%$ in the Netherlands, $27.7 \%$ in France $27.7 \%$, and so on.

In 2011, under the leadership of WHO, more than 190 countries adopted an action plan and road map with policy options focusing on four modifiable risk behaviors; as a result, preventing and controlling NCDs for 2013-2020 (WHO, 2014b). This plan aims to reduce by $25 \%$ the global number of premature deaths by achieving the nine targets presented in Table 2 .

Table 2

Global Targets for Prevention and Control of NCD

Global Targets for Prevention and Control of $N C D$
1 A $25 \%$ relative reduction in risk of premature mortality from cardiovascular diseases, cancer, diabetes,
or chronic respiratory diseases.
At least $10 \%$ relative reduction in the harmful use of alcohol, as appropriate, within the national context.
A $10 \%$ relative reduction in prevalence of insufficient physical activity.
A 30\% relative reduction in mean population intake of salt/sodium.
5 A $30 \%$ relative reduction in prevalence of current tobacco use in persons over 15.
6 A 25\% relative reduction in the prevalence of high blood pressure or not increasing the percentage of
those with high blood pressure according to national circumstances.
Halt the rise in diabetes and obesity.
8 At least $50 \%$ of eligible people receive drug therapy and counseling (including glycemic control) to
prevent heart attacks and strokes.
An $80 \%$ availability of affordable basic technologies and essential medicines, including generics, required
to treat major NCDs in both public and private facilities.

Source: WHO (2014b).

For the performance of each EU member nation's health system, the EU member nation must respond by developing and applying nine policies adequate for each target and having:

- an operational NCD unit/branch or department within the Ministry of Health, or equivalent 
- an operational multi-sector national policy, strategy, or action plan that integrates several NCDs and shared risk factors

- an operational policy, strategy or action plan to reduce the harmful use of alcohol

- an operational policy, strategy or action plan to reduce physical inactivity and/ or promote physical activity

- an operational policy, strategy or action plan to reduce the burden of tobacco use

- an operational policy, strategy or action plan to reduce unhealthy and/or promote healthy diets

- evidence-based national guidelines/protocols/standards for the management of major NCDs through a primary care approach

- an NCD surveillance and monitoring system in place to enable reporting against the nine global NCD targets

- a national, population-based cancer registry.

Figure 2 shows the situation in EU countries. The number of applied health policies is found to be different. In countries with a greater number of applied health policies (8), the probability of dying prematurely is lower (11-12\%).

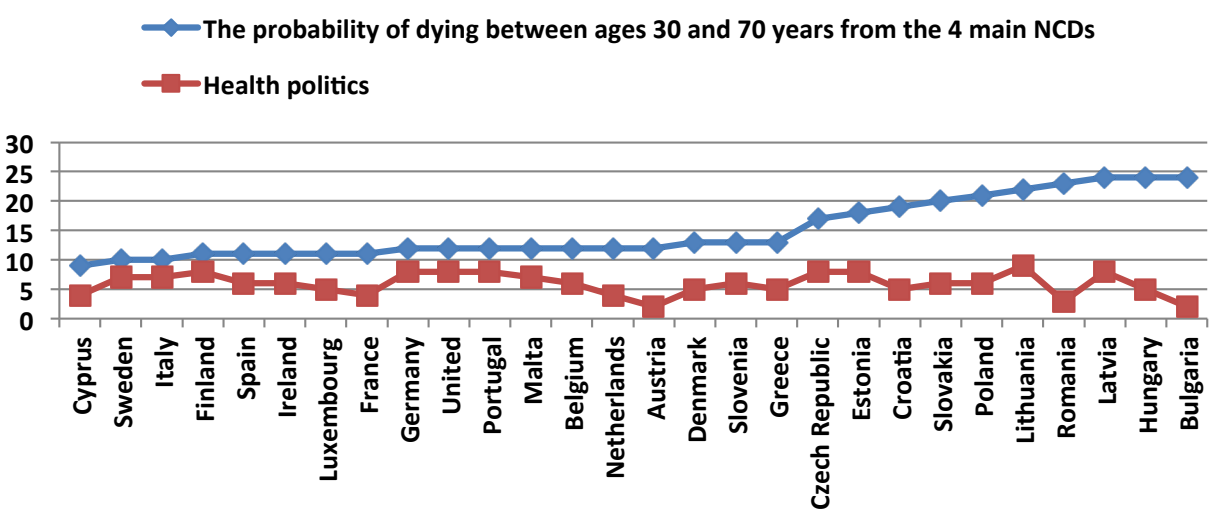

Figure 2. Association between the probability of premature death and the number of applied health policies in the EU.

Bulgaria has the least number of applied policies (2), and Lithuania has the most (9).

According to WHO, only two policies are implemented effectively in Bulgaria: having evidence-based national guidelines/protocols/standards for managing major NCDs through a primary care approach and a national, population-based cancer registry (WHO, 2014c). 


\section{Conclusion}

Implementing more health policies at a national level is essential for solving problems in terms of chronic non-communicable diseases in Bulgaria, which has the highest probability of premature death and the lowest number of policies compared to other EU countries.

\section{References}

Bonita, R., Magnusson, R., Bovet, P., Zhao, D., Malta, D. C., Geneau, R., ... Lancet NCD Action Group. (2013). Country actions to meet UN commitments on non-communicable diseases: A stepwise approach. Lancet, 381(9866), 575-584. http://dx.doi.org/10.1016/S01406736(12)61993-X

Borisov, V. (1990). Social hygiene methodology (Master's thesis). Medicina i Fizkultura, Sofia, Bulgaria.

Grancharova, G., \& Alexandrova-Jankulovska, S. (Eds.). (2015). Social medicine. Bulgaria, Pleven: IC MU.

National Center of Public Health and Analyses. (2015). Annual public health statistics. Bulgaria. Retrieved from http://ncphp.government.bg/en

World Health Organization. (2010). Global status report on noncommunicable diseases 2010. Geneva, Switzerland: Author. Retrieved from http://www.who.int/nmh/publications/ncd report2010/en/

World Health Organization. (2014a). Global status report on noncommunicable diseases 2014. Geneva, Switzerland: Author. Retrieved November 12, 2015 from http://www.who.int/nmh/ publications/ncd-status-report-2014/en/

World Health Organization. (2014b). Global action plan for the prevention and control of noncommunicable diseases 2013-2020. Geneva, Switzerland: Author. Retrieved from http:// www.who.int/nmh/publications/ncd-action-plan/en/

World Health Organization. (2014c). Noncommunicable Diseases Country Profiles 2014 (p. 39). Geneva, Switzerland: Author. Retrieved from http://www.who.int/nmh/countries/en/

World Health Organization. (2015). Health 2015 from millennium development goals to sustainable development goals (pp. 131-151). Geneva, Switzerland: Author. Retrieved from http://www. who.int/gho/publications/mdgs-sdgs/en/ 